\section{Zystische Fibrose: neue antibiotische Option}

— Seit April diesen Jahres kann bei Zystischer Fibrose (CF) ein neues inhalatives Antibiotikum verordnet werden: Aztreonam-Lysin $\left(\right.$ Cayston $\left.^{\circledR}\right)$, das in einer gemeinsamen Verpackung mit dem eigens für Cayston ${ }^{\circledR}$ entwickelten ALTERA ${ }^{\circledR}$-Verneblersystem angeboten wird. Wie Prof. Dr. Dieter Adam, München, erklärte, ist AztreonamLysin ein sogenanntes Monobactam aus der Reihe der Betalactam-Antibiotika. Das antimikrobielle Wirkspektrum umfasst ausschließlich gramnegative Erreger, sodass es bei schweren bakteriellen Infektionen unklarer Genese praktisch nur in Kombination mit anderen, hauptsächlich gegenüber grampositiven Mikroorganismen wirksamen Antibiotika zum Einsatz kommt. Dies ist ein Grund, weshalb Aztreonam im Laufe der letzten Jahre immer seltener angewendet wurde, wodurch es nach Adam zu einem „echten Reserveantibiotikum” geworden ist. Aztreonam ist nicht magensäurestabil und kann deshalb nur parenteral oder lokal, beispielsweise als Lysin zur Inhalation, verabreicht werden. Zur inhalativen Therapie bronchopulmonaler Infektionen (vorzugsweise verursacht durch Pseu- domonaden) bei Patienten mit CF eignet sich Aztreonam-Lysin auch allein, also ohne Kombination mit anderen Antibiotika. Aztreonam ist stabil gegenüber Betalactamasen und entwickelt keinerlei Kreuzallergie zu Penicillinen, Cephalosporinen oder Carbapenemen. Es kann anstelle der bei dieser Indikation oft eingesetzten Aminoglykoside, wie beispielsweise Tobramycin, angewendet werden. Wie Adam erläuterte, ist dies bei den immer häufiger auftretenden Resistenzen gegenüber Aminoglykosiden von Vorteil.

Die Europäische Arzneimittelagentur berichtete in einer Zusammenfassung des Europäischen Öffentlichen Beurteilungsberichts, dass laut des Ausschusses für Humanarzneimittel „ein bislang ungedeckter medizinischer Bedarf an neuen Antibiotika für Patienten mit Mukoviszidose besteht, da viele dieser Patienten bereits vor Erreichen des Erwachsenenalters eine Resistenz gegen andere Antibiotika entwickelt haben".

Das Präparat wurde nach Herstellerangaben „unter Auflagen” zugelassen, dass heißt, dass weitere Nachweise erwartet werden,

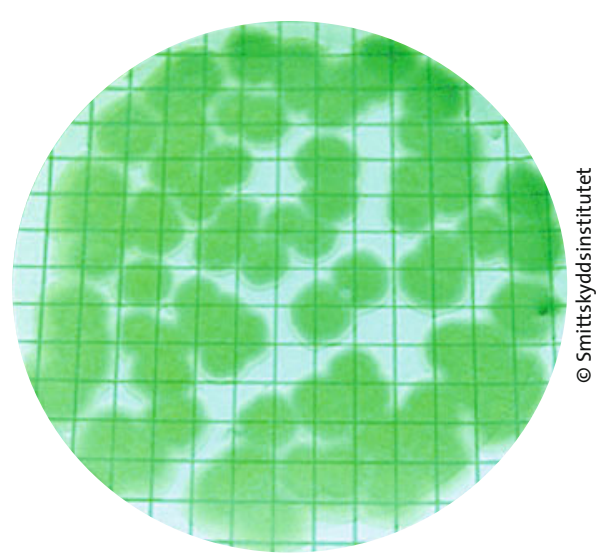

Kolonien von Pseudomonas aeruginosa

die darüber Aufschluss geben, „ob die in den Kurzzeitstudien festgestellten Vorteile über mehrere Behandlungszyklen hinweg erhalten bleiben". Dann sollen auch mehr Informationen über die Langzeitsicherheit und die Resistenzentwicklung gegenüber Pseudomonas aeruginosa vorliegen. jset

Pressekonferenz „Cayston ${ }^{\circledR}$ für CF-

Patienten - eine Therapielücke wird geschlossen" anlässlich des 51. Kongresses der Deutschen Gesellschaft für Pneumologie und Beatmungsmedizin (DGP). Hannover, 18. März 2010. Veranstalter: GILEAD, Martinsried

\section{Pneumokokken - was für ältere Kinder gilt}

— Die STIKO hat ihre Empfehlungen zu Indikationsimpfungen geändert: Pädiater sollen gefährdete Kinder von zwei bis fünf Jahren mit einer Konjugatvakzine gegen Pneumokokken impfen. Die bisher empfohlene 23-valente Polysaccharidvakzine wird durch einen Konjugat-Impfstoff abgelöst. Als einziger Pneumokokken-Konjugatimpfstoff ist für Kinder von zwei bis fünf Jahren die 13 -valente Vakzine Prevenar13 ${ }^{\circledR}$ zugelassen. Darin ließen sich durch Konjugation der Pneumokokken-Kapselpolysaccharide an ein Trägerprotein Immunogenität und Schutzwirkung erhöhen, erklärte Prof. Dr. Ulrich Heininger aus Basel. Die im Vergleich zum Polysaccharid-Impfstoff fehlenden Serotypen seien für Zwei- bis Fünfjährige nicht so bedeutsam. Der Effekt durch Prevenar $13^{\circledR}$ gegen Serotyp $6 \mathrm{~A}$ allerdings sei für sie epidemiologisch wichtig, sagte Heininger. Weitere Vorteile des Konjugatimpfstoffs: bessere Verträglichkeit, einfache Handhabung durch einmalige Impfung sowie keine verminderte Immunantwort bei wiederholter Impfung - ein häufiges Problem bei Polysaccharid-Vakzinen. Gefährdete Kinder profitieren besonders von dem 13-valenten Impfstoff, wenn sie ungeimpft sind oder in den ersten beiden Lebensjahren nur die 7-valente Vakzine erhalten hatten. Ebenfalls wirksam - obgleich etwas weniger - ist die zusätzliche Impfung mit dem 13-valenten Impfstoff, wenn die Kinder in der Grundimmunisierung plus Booster den 10-valenten Impfstoff verabreicht bekamen.

Der Arzt müsse für eine Indikationsimpfung nach dem zweiten Lebensjahr das individuelle Risiko einschätzen, erläuterte der Kinderarzt Jörn Voigt aus Groß-Umstadt. Zu berücksichtigen seien einerseits chronische Krankheiten wie Asthma bronchiale, neurologische Störungen wie Zerebralparesen oder Epilepsie, Diabetes und andere Stoffwechseldefekte, Herz-Kreislauf- sowie Nieren-Krankheiten. Andererseits sollten
Kinder mit angeborenen oder erworbenen Immundefekten mit Restfunktion der T- oder B-Zellen, etwa bei Tumoren, HIV-Infektionen oder nach Knochenmarktransplantation geimpft werden. Für sinnvoll hält Voigt es auch, bisher ungeimpfte Kita-Kinder generell zu impfen.

Der 13-valente Impfstoff ist zugelassen für Kinder ab sechs Wochen bis zu fünf Jahren. Indikation ist die Vorbeugung invasiver Erkrankungen, Pneumonie und akuter Otitis media, die durch die Serotypen 1, 3, 4, 5, 6A, $6 \mathrm{~B}, 7 \mathrm{~F}, 9 \mathrm{~V}, 14,18 \mathrm{C}, 19 \mathrm{~A}, 19 \mathrm{~F}$ und $23 \mathrm{~F}$ von Streptococcus pneumoniae verursacht werden. Dabei rät die STIKO zum 3+1-Schema: Zeitgleich mit anderen für Säuglinge empfohlenen Impfungen wird die Grundimmunisierung mit drei Impfdosen im Alter von zwei, drei und vier Monaten plus Booster im Alter von 11 bis 14 Monaten vorgenommen.

Pressegespräch „Prevenar 13 - STIKO-Empfehlungen: Was gibt es Neues?" anlässlich der 106. Jahrestagung der DGKJ. Potsdam, 17. September 2010. Veranstalter Pfizer, Berlin 